\title{
BMJ Open Development of a computerised neurocognitive battery for children and adolescents with HIV in Botswana: study design and protocol for the Ntemoga study
}

\author{
J Cobb Scott (D) , ${ }^{1,2}$ Amelia E Van Pelt, ${ }^{3,4}$ Allison M Port, ${ }^{1}$ Lucky Njokweni, ${ }^{1}$ \\ Ruben C Gur, ${ }^{1}$ Tyler M Moore, ${ }^{1}$ Onkemetse Phoi, ${ }^{5}$ Ontibile Tshume, ${ }^{5}$ \\ Mogomotsi Matshaba, ${ }^{5,6}$ Kosha Ruparel, ${ }^{1}$ Jennifer Chapman, ${ }^{3}$ \\ Elizabeth D Lowenthal ${ }^{3,4}$
}

To cite: Scott JC, Van Pelt AE, Port AM, et al. Development of a computerised neurocognitive battery for children and adolescents with HIV in Botswana: study design and protocol for the Ntemoga study. BMJ Open 2020;10:e041099. doi:10.1136/ bmjopen-2020-041099

- Prepublication history for this paper is available online. To view these files, please visit the journal online (http://dx.doi. org/10.1136/bmjopen-2020041099).

Received 29 May 2020 Revised 10 July 2020 Accepted 13 July 2020

D) Check for updates

(C) Author(s) (or their employer(s)) 2020. Re-use permitted under CC BY-NC. No commercial re-use. See rights and permissions. Published by BMJ.

For numbered affiliations see end of article.

\section{Correspondence to}

Dr J Cobb Scott;

scott1@pennmedicine.upenn. edu

\section{ABSTRACT}

Introduction Neurodevelopmental delays and cognitive impairments are common in youth living with HIV. Unfortunately, in resource-limited settings, where HIV infection impacts millions of children, cognitive and neurodevelopmental disorders commonly go undetected because of a lack of appropriate assessment instruments and local expertise. Here, we present a protocol to culturally adapt and validate the Penn Computerized Neurocognitive Battery (PennCNB) and examine its validity for detecting both advanced and subtle neurodevelopmental problems among school-aged children affected by HIV in resource-limited settings. Methods and analysis This is a prospective, observational cohort study. The venue for this study is Gaborone, Botswana, a resource-limited setting with high rates of perinatal exposure to HIV and limited neurocognitive assessment tools and expertise. We aim to validate the PennCNB in this setting by culturally adapting and then administering the adapted version of the battery to 200 HIV-infected, 200 HIV-exposed uninfected and 240 HIV-unexposed uninfected children. A series of analyses will be conducted to examine the reliability and construct validity of the PennCNB in these populations.

Ethics and dissemination This project received ethical approval from local and university Institutional Review Boards and involved extensive input from local stakeholders. If successful, the proposed tools will provide practical screening and streamlined, comprehensive assessments that could be implemented in resourcelimited settings to identify children with cognitive deficits within programmes focused on the care and treatment of children affected by HIV. The utility of such assessments could also extend beyond children affected by HIV, increasing general access to paediatric cognitive assessments in resource-limited settings.

\section{BACKGROUND}

Approximately 1.8 million children worldwide are living with HIV. ${ }^{1}$ Although the
Strengths and limitations of this study

- This protocol aims to culturally adapt and validate a computerised neurocognitive battery in 200 HIVinfected, 200 HIV-exposed uninfected and 240 HIVunexposed uninfected children in Botswana.

- This protocol involves the local expertise of patients and stakeholders to inform cultural adaptations and translations.

- The study proposes to use a multimethod framework for validation of the neurocognitive battery, including reliability, structural validity, concurrent validity and criterion validity.

- The study also aims to develop a brief screening measure to identify children in need of further neurocognitive evaluation.

- The study is limited by the lack of a gold-standard cognitive assessment in Botswana to which we can compare.

incidence of HIV-related encephalopathy decreased with the introduction of combined antiretroviral therapies (cART), ${ }^{2}{ }^{3}$ children and adolescents living with HIV still evidence neurodevelopmental delays and cognitive dysfunction. Cognitive deficits are primarily found in attention, information processing speed, episodic memory, executive functioning and psychomotor functioning. ${ }^{4-12}$ Deficits are greater in children with a history of higher disease severity, suggesting residual effects of HIV-associated cerebral injury. ${ }^{11} 13$ However, deficits are apparent even when the disease is well-managed ${ }^{41011} 14$ and are associated with disease progression, functional limitations and future cognitive decline. ${ }^{11} 15$ Moreover, initiation of cART may halt the progression of neurocognitive dysfunction 
but does not reverse existing dysfunction. ${ }^{16-19}$ There is also evidence that HIV-exposed uninfected children (HEU) suffer from more neurodevelopmental delays than HIV-unexposed uninfected children (HUU). ${ }^{11}$ 20-22

Taken together, it is clear that early, accurate detection of neurocognitive deficits is crucial for effective clinical, educational and rehabilitative care. Accurate assessments are particularly critical in areas with a high HIV prevalence, in resource-limited settings where exposures to HIV impact large numbers of children and in settings in which neurobehavioural disorders commonly go undetected. ${ }^{23}$ However, few locally validated cognitive assessments exist in resource-limited settings, which presents significant challenges for assessing cognition in adults and children with HIV.

Screening measures have been created to provide brief assessments for HIV-associated neurocognitive disorders, although most do not possess adequate diagnostic precision. ${ }^{24}$ Thus, it is recommended that a full neuropsychological battery assessing multiple domains of cognitive functioning be used to detect HIV-associated neurocognitive dysfunction. ${ }^{25}$ These batteries provide critical information about the pattern and severity of neurocognitive deficits and aid in disease management and understanding functional limitations. However, most neuropsychological batteries are proprietary, expensive and resource intensive. Most involve 3-5 hours of testing, with highly specialised training required in administration, interpretation and scoring. Furthermore, such batteries are predominantly administered by paper and pencil, raising the risk of errors in administration and scoring and creating challenges for data entry, management and rapid turnaround of results. Thus, these testing batteries are not feasible in many settings, including those with limited resources.

Modern computerised neurocognitive testing allows shorter testing times while offering sophisticated features unavailable to traditional neuropsychological tests, such as automatic, precise recording of response times and accuracy. The Brain Behavior Lab at the University of Pennsylvania has developed the Penn Computerized Neurocognitive Battery (PennCNB), ${ }^{26-28}$ a publicly available collection of 'neurobehavioral probes' designed to assess psychological constructs (eg, working memory) by way of their underlying brain networks. PennCNB tests have been subjected to an iterative validation process to evaluate their psychometric properties ${ }^{26} 27$ and normed in multiple populations. ${ }^{28-31}$ Importantly, PennCNB tests have been validated with functional neuroimaging as eliciting the recruitment of specific brain systems, ${ }^{32-35}$ making them useful as potential biomarkers of brain dysfunction. ${ }^{28}$ The cognitive domains assessed by conventional neuropsychological tests ${ }^{36}$ are readily replicated by the PennCNB, reinforcing its potential validity in individuals living with HIV. The PennCNB has been applied in several contexts, including large-scale genomic studies, ${ }^{28-30}$ longitudinal research, ${ }^{37} 38$ intervention studies ${ }^{39}$ and even in space flight. ${ }^{40}{ }^{41}$ Importantly, the measures are well-tolerated by children and have been used for several specialised paediatric populations, including children with 22q11.2 deletion syndrome ${ }^{42-45}$ and chronic kidney disease ${ }^{46}$ as well as the landmark Philadelphia Neurodevelopmental Cohort study, a sample of 9500 youth aged 8-21 years. ${ }^{47}$ Critically, the PennCNB has been culturally adapted in western and non-western cultures and translated into 12 languages, including a Nguni African language (isiXhosa). ${ }^{3748-52}$

The current research protocol focuses on Botswana, a resource-limited setting with scarce neurocognitive or neurodevelopmental assessment tools and expertise. As the first country in Africa to provide free antiretroviral therapy in the public sector to all citizens living with HIV, Botswana has a large ageing population of children and adolescents with perinatally acquired HIV. ${ }^{53}$ In the proposed project, we aim to establish the validity, reliability and feasibility of administering the PennCNB in Botswana to assess neurocognitive functioning in children living with HIV.

In addition, we aim to develop a screening measure to identify which children would benefit from assessment with the PennCNB by adapting the Pediatric Symptom Checklist (PSC) ${ }^{54}$ a 3-5 min parent-report questionnaire validated for detection of psychosocial risk in children in several cultural contexts, including in Botswana by members of our team. ${ }^{55-57}$ We aim to enhance its sensitivity for identifying children with neurocognitive difficulties through adaptations, including adding questions about domains important for children with HIV-related cognitive dysfunction, such as academic functioning and self-direction.

\section{METHODS}

\section{Participants}

Three groups of participants will be selected (figure 1). We will enrol 200 children with HIV (HIV+), 200 HEU and $240 \mathrm{HUU}$, all between 7 and $<18$ years old. We focus on school-aged children because some CNB subtests are less suitable for preschool-aged children, and most prior work has been conducted in school-aged

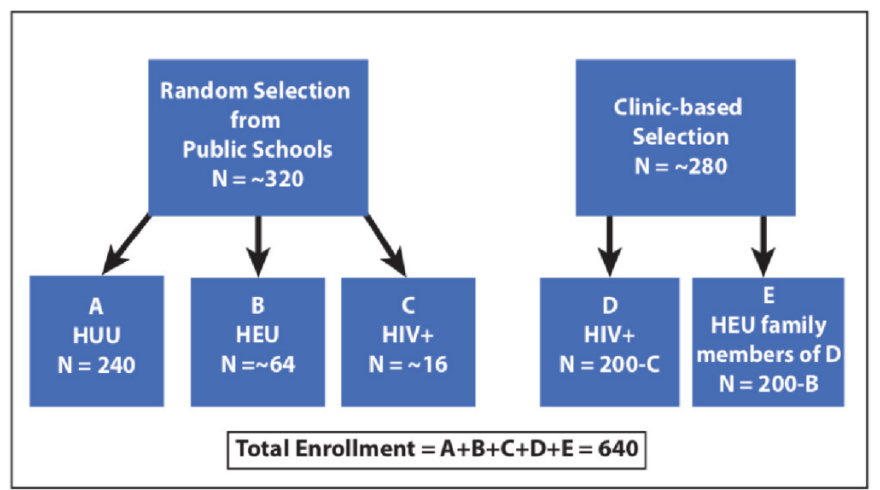

Figure 1 Study flowchart. Figure does not include the additional participants being enrolled as cases for the gold standard assessment. HEU, HIV exposed uninfected; HIV+, children with HIV; HUU, HIV unexposed uninfected. 
children, enabling a more extensive knowledge base for comparison. HIV+ youth will be randomly selected from eligible youth in care at the Botswana-Baylor Children's Clinical Centre of Excellence (COE) in Gaborone. Youth with HIV will be predominantly perinatally infected, and likely mode of infection for each participant will be recorded. We will enrol children randomly selected from local public schools in Gaborone, Botswana until 240 HUU children are enrolled. Randomisation will be stratified by age bands (ie, $7 / 8,9 / 10$, $11 / 12,13 / 14,15-17)$ for robust normative comparisons. Based on local seroprevalence data, it is estimated that $\sim 5 \%$ of school-going youth will be HIV+ and $\sim 20 \%$ will be HEU. HIV status and HIV exposure status will be unknown at the time that school-based participants are consented, though this information will be elicited. Students recruited from schools and HIV+ children enrolled in the clinic will be randomly selected from among all students/patients in an age stratum to achieve a minimum of 30 in each stratum. HEU children who are not part of the randomly selected school sample will be selected by recruiting family members of clinical patients to enrol $200 \mathrm{HEU}$ children.

Parents of enrolled children will privately complete surveys that will include HIV and HIV exposure status.
Enrolment data will be uploaded daily to the data coordinating centre. A record will be kept (age, sex, reason for exclusion) of all individuals who were selected for enrolment but not enrolled.

\section{Neurocognitive assessment}

The core paediatric version of the PennCNB comprises 14 tests assessing five neurobehavioral domains. ${ }^{31}$ For the proposed study, we selected 13 tests from this battery and from our prior HIV work, as shown in figures 2 and 3 and described later. Tests were selected for inclusion based on the following criteria: (1) ability to assess the cognitive domains impacted in paediatric HIV and in utero HIV or antiretroviral therapy exposure ${ }^{4-105859}$; (2) suitability for large-scale administration; (3) suitability for resourcelimited settings; (4) suitability for translation and adaptation across cultures (eg, low language demands, limited unfamiliar stimuli); and (5) demonstrated sensitivity to individual differences and mild impairments. To enhance reliability and sensitivity, we use more than one test within each domain. All tests except the Penn Conditional Exclusion Test (PCET) include practice periods to ensure task engagement and comprehension.

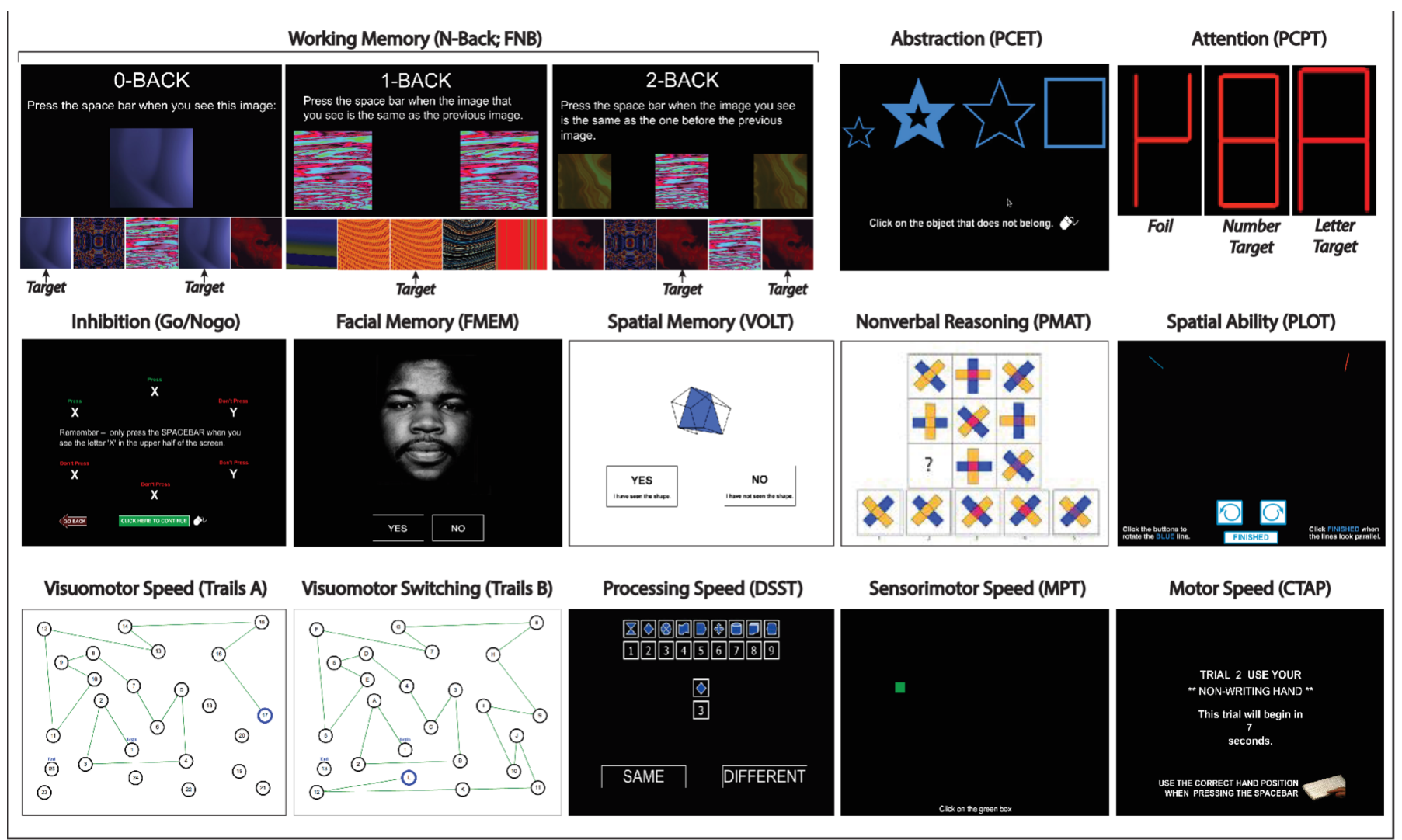

Figure 2 Tests selected from the Penn Computerized Neurocognitive Battery (PennCNB), English version. Stimuli used with permission from Dr Ruben Gur, lead author of the PennCNB. Image used for the FMEM is used as a stimulus and is not from a participant. Actors in these photos consented for their subsequent use without limitation. CTAP, Penn Finger Tapping Test; DSST, Penn Digit Symbol Substitution Test; FMEM, Penn Facial Memory Test; FNB, Fractal N-Back; MPT, Motor Praxis Test; PCET, Penn Conditional Exclusion Test; PCPT, Penn Continuous Performance Test; PLOT, Penn Line Orientation Test; PMAT, Penn Matrix Analysis Test; VOLT, Visual Object Learning Test. 


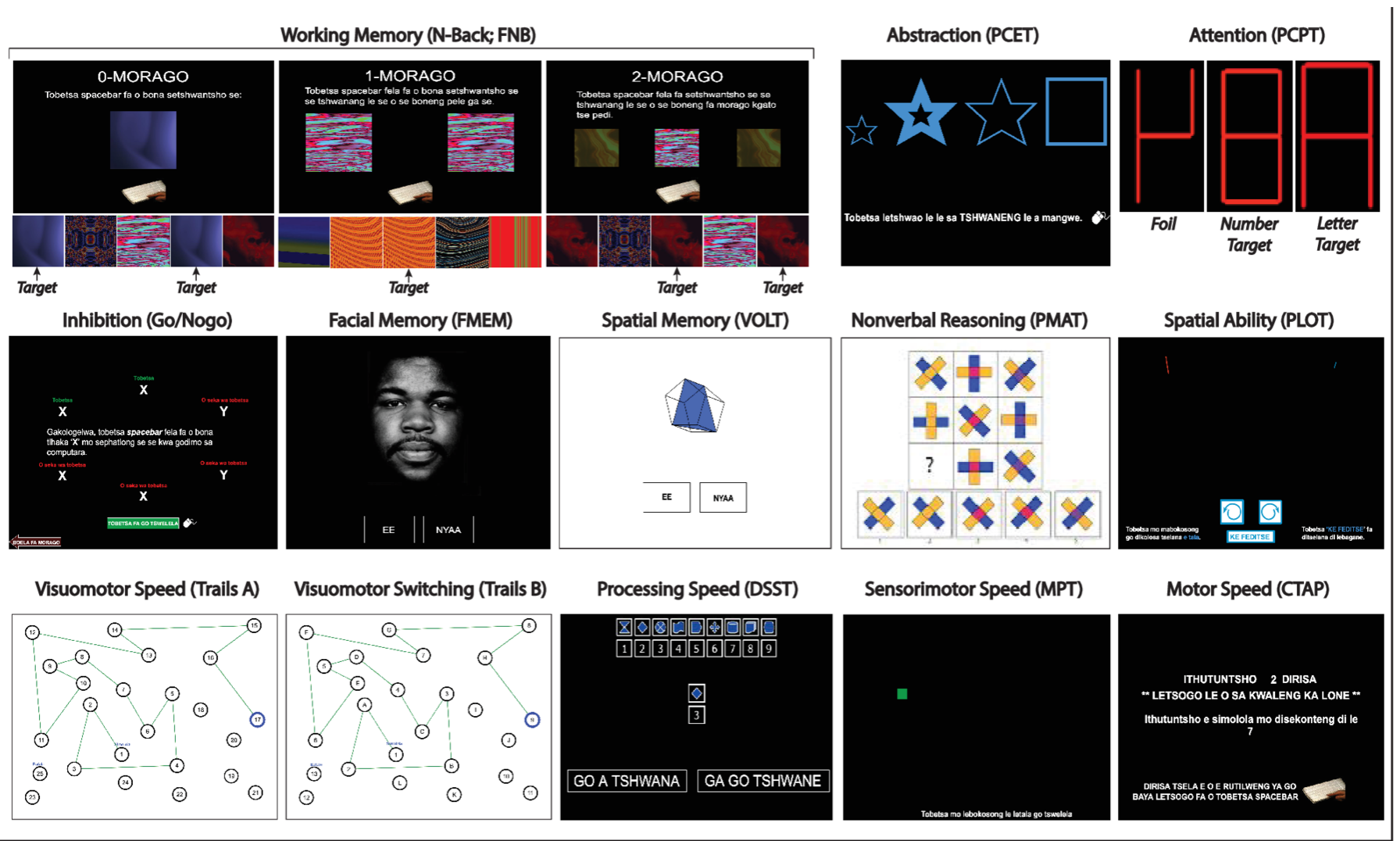

Figure 3 Tests selected from the Penn Computerized Neurocognitive Battery (PennCNB), Setswana Version. Stimuli used with permission from Dr Ruben Gur, lead author of the PennCNB. Image used for the FMEM is used as a stimulus and is not from a participant. Actors in these photos consented for their subsequent use without limitation. CTAP, Penn Finger Tapping Test; DSST, Penn Digit Symbol Substitution Test; FMEM, Penn Facial Memory Test; FNB, Fractal N-Back; MPT, Motor Praxis Test; PCET, Penn Conditional Exclusion Test; PCPT, Penn Continuous Performance Test; PLOT, Penn Line Orientation Test; PMAT, Penn Matrix Analysis Test; VOLT, Visual Object Learning Test.

Attention and processing speed

Penn Continuous Performance Test $(\mathrm{PCPT})^{60}$; Digit Symbol Substitution Test (DSST) ${ }^{61}$; and Penn Trailmaking Test, Part A (TMTA). The PCPT presents 7-segment displays $(1 / \mathrm{s})$ and requires participants to respond when the segments form a number (first $1.5 \mathrm{~min}$ ) or letter (next $1.5 \mathrm{~min}$ ). The number of true positive responses reflects accuracy, and the median response time for true positives reflects attention speed. The DSST presents a reference set of digit-symbol pairs and a target digit-symbol pair. Participants are instructed to quickly indicate if the target pair matches one of the digit-symbol pairs in the reference set. The TMTA is a computerised analogue of the classic Trailmaking test that measures visual attention and processing speed, requiring participants to connect a sequence of numbers in sequential order. ${ }^{62}$

\section{Episodic memory}

Penn Face Memory Test (FMEM) ${ }^{63}$ and Visual Object Learning Test (VOLT). ${ }^{64}$ The FMEM presents 20 faces to remember, while the recall portion shows these target faces mixed with 20 distractors equated for age, gender and race. The version of the FMEM proposed in this study uses a multiracial assortment of faces. The VOLT shows participants a series of 10 three-dimensional Euclidean shapes. They are then shown a mixture of shapes they have seen and 10 novel shapes, and are asked to decide whether they have seen each shape before.

\section{Executive functioning}

PCET $^{6566}$; Fractal N-Back Test $(\mathrm{FNB})^{6768}$;Penn Trailmaking Test, Part B (TMTB); and Penn Go/Nogo (GNG). ${ }^{38}$ The PCET is a measure of abstraction and concept formation by hypothesis-testing, in which participants are presented with four objects and asked to select the one that does not belong, based on a sorting principle (eg, size). After a set number of successive correct responses, the sorting principle is changed (as in the Wisconsin Card Sorting Test). The FNB measures working memory by presenting a series of figures (fractals) and requiring the participant to identify visual repetitions occurring ' $n$ ' trials preceding the current stimulus. The TMTB is similar to the TMTA, but requires participants to alternate between letters and numbers (ie, $1 \rightarrow A \rightarrow 2 \rightarrow B$ ), engaging set-switching abilities. ${ }^{96}$ The GNG is a measure of impulse control that requires participants to respond to a series of targets (an ' $\mathrm{X}$ ' in the upper half of the screen) and inhibit responding to low-frequency non-targets (' $\mathrm{X}$ ' in the lower half of the screen or a ' $\mathrm{Y}$ ' anywhere). The test induces participants to develop a response tendency and then interrupts that 
tendency with intermittent non-targets to which participants have to inhibit responding.

\section{Complex cognition}

Penn Line Orientation Test $(\mathrm{PLOT})^{70}$ and Penn Matrix Analysis Test (PMAT).$^{71}$ The PLOT presents two angled lines. Participants click a button to rotate one line until they believe it has the same angle as the other. The PMAT is a measure of non-verbal reasoning that uses the same principles as Raven's progressive matrices ${ }^{72}$ consisting of matrices requiring reasoning by geometric analogy and contrast principles.

\section{Sensorimotor speed}

Finger Tapping Test $(\mathrm{CTAP})^{73}$ and Motor Praxis Test (MPT). The CTAP measures how quickly the participant can press the spacebar using the index finger. The MPT requires moving the mouse and clicking on a green square that disappears after the click. The square gets increasingly small and appears in unpredictable locations.

\section{Cultural adaptation and translation}

For the Setswana version of the CNB and clinical interview materials, we followed WHO translation guidelines. The process of cultural and language adaptations involved a formal procedure that we have previously applied. ${ }^{74} 75$ This involved translation by a professional, followed by: (1) iterations of back-translating, resolving discrepancies and re-translating (when necessary); (2) piloting of tests and items; (3) discussions about challenging concepts; and (4) further modifications of the instruments to select the most linguistically and conceptually appropriate Setswana terminology. Concurrently, English terminology was adapted to be locally appropriate.

\section{Patient and public involvement}

At the initiation of the study, the principal investigators (EDL and JCS) met with clinicians and staff at the Baylor-Botswana COE in Gaborone and with other local stakeholders. This stakeholder group consisted of representatives from the Botswana Ministry of Basic Education and Ministry of Health and Wellness, physicians, teachers from area schools, school counsellors, social workers, nurses, psychologists, representatives from UNESCO, three young adults who were patients in the clinic cohort, and a parent of a clinic child with cognitive challenges. This multidisciplinary group of stakeholders was convened for 2 days to provide feedback on concepts and stimuli, offer thoughtful back-translation of the research instruments, assist with and discuss conceptual and linguistic discrepancies occurring during translation/ back-translation, and promote the substantive involvement of the communities.

The stakeholder group offered important feedback to guide adaptations. First, they suggested that some older adolescent participants might prefer to receive the assessments in English, since English is used for higher level instruction in schools. Notably, this feedback was supported by our pilot testing (see later), in which two older participants asked to receive assessments in English. Thus, we created equivalent versions of assessments in English and Setswana. Second, they suggested a greater racial diversity of faces for the FMEM, which we adapted to address this feedback. Third, they suggested (and we developed) a formal practice period for gaining familiarity with the computers since many participants might not have used computers previously. Fourth, they suggested that certain words such as those for computer components (eg, screen, mouse) should be explained by study staff since there are limited appropriate Setswana words; standardised methods for these explanations were developed. Finally, the group suggested simpler ways of conveying several ideas that were initially translated into Setswana in a more formal or literal fashion.

The stakeholder group also chose the study name, 'Ntemoga'. Ntemoga is a multifaceted Setswana word with meanings that are commonly translated as 'cognition', but also: 'know me inside and out', 'acknowledge that I matter', 'notice me', 'take me seriously' and 'know my needs'.

\section{Pilot sample}

After completing adaptation and training, JCS and AMP supervised piloting of the tools to finalise procedures and get feedback from participants and assessors. Local assessors administered the battery to 10 children and adolescents with HIV from the clinic, including five boys and five girls distributed in ages between 7 and 17. This pilot sample assisted with additional minor modifications to instructions and study procedures.

\section{Procedures for validation study}

Parents of participants will provide demographic and health information and school reports. History of hospitalisations, nervous system disorders, surgeries requiring anaesthesia and current medication use will be collected. For HIV+ participants, we will record WHO clinical staging, WHO T-staging, nadir CD4+ T-lymphocyte count (CD4), and most recent CD4 and viral load. Participants will receive compensation for participating, with amounts determined by the national Health Research Development Council (equivalent to about US $\$ 4 /$ participant per study visit).

Participants will be administered the PennCNB on laptops (MacBooks) by proctors with extensive remote and on-site training by PennCNB staff. Each facility provides a secluded and quiet room with adequate space. Facilities and procedures were verified during site visits by JCS, EDL, AMP, and monitored on an ongoing basis by local study staff. Proctors were trained to note protocol issues in designated fields and provide information necessary for determining the validity of results for each test separately and for the battery as a whole. All data from the validation sample will be used for validation procedures discussed later under the Analyses section. 


\section{'Gold standard' assessments}

To assess the criterion validity of the PennCNB, in a subset of enrolled participants, we will evaluate its ability to identify children who are classified as cognitively impaired or unimpaired based on the best available local assessments. Participants for this subset will be drawn from two sources. HIV+ participants aged 10-17 years in the randomly selected clinic cohort will be evaluated by a clinical psychologist for possible inclusion. To supplement the randomly selected clinic patients, patients with HIV previously identified as having neurocognitive impairments will also be selected for re-evaluation to ensure adequate numbers of 'impaired' participants. We will select patients until 45 'cases' and 23 'controls' are identified.

Due to limited validated cognitive assessment tools in Botswana, 'gold standard' assessments use a team-based approach to comprehensively evaluate cognitive abilities and daily functioning of this subset. A local clinical psychologist will conduct an evaluation, consisting of: (1) an intake assessment, with in-depth qualitative interviews with the child and parent and review of school records; (2) Montreal cognitive assessment, ${ }^{76}$ a 30 -question tool designed to assess cognitive ability in eight domains (orientation, short-term memory, executive function, language, abstraction, attention, animal naming and visuoconstructional); and (3) Draw-a-Person Test, measuring cognitive maturity and mental age. Through comprehensive review of the data, the clinical psychologist will preliminarily classify a participant as a case (ie, with clinically significant cognitive impairment) or a control (ie, without clinically significant cognitive impairment). The preliminary classifications will be discussed by a group of four clinicians with local and international expertise in paediatrics, neuropsychology and HIV, who will review data to determine whether each participant should be classified as a case, with cognitive impairments impacting daily functioning. If there is a lack of consensus, the participant will be deemed 'unclassifiable' and not included among cases and controls for analysis. To ensure that the randomly selected sample remains representative of the underlying clinical population, cases not drawn from the 200 randomly selected clinic patients will not be included in other analyses described later.

\section{Screening assessment development- the 'Ntemoga Screener'}

Since the PennCNB takes over an hour to administer and requires trained proctors, we also aim to develop a simpler screening tool to prioritise children who are most likely to benefit from PennCNB screening. First, the PSC will be administered to each participant's parent to assess participant psychosocial difficulties. ${ }^{54}$ Notably, the PSC displays high internal consistency, moderate to high test-retest reliability ${ }^{77} 78$ and moderate to strong correlations with ratings from psychiatric interviews. ${ }^{547980}$ The PSC also shows promise for identifying children in need of further evaluation for neurocognitive dysfunction. ${ }^{81}$ Thus, we aim to enhance its sensitivity for that purpose through adaptations, including removal of items that are less discriminative for prediction of cognitive dysfunction and addition of questions querying relevant areas such as academic functioning and self-direction. This new 'Ntemoga Screener' will combine items from the PSC with additional items selected for their likely sensitivity to cognitive dysfunction and need for further evaluation. Use of this tool is similar to 'pipeline optimisation' in machine learning, ${ }^{82}$ in which, for example, a subsample of patients might be 'ruled out' from the very beginning as not having an illness. This is useful in statistical analysis but also has important practical implications, such as obviating the need for further medical assessment.

\section{Analyses}

\section{Data cleaning}

Response patterns for individual tests will be examined for subject-related problems (eg, extended periods of inattention, participant misunderstanding). Thresholds for whether to flag a test for validity problems vary by test. Flagged individual tests will be removed from analyses, so it is possible for some participants to only have data for some tests. Missing data will be handled using pairwise and (then) listwise deletion in all analyses so that effects of missing data handling can be compared.

\section{Reliability}

Reliability will be assessed with measures of internal consistency (Cronbach's $\alpha$ and McDonald's $\omega_{\mathrm{H}}{ }^{83}$ ) and test-retest reliability. To assess test-retest reliability, 50 participants will repeat testing in approximately 12 weeks. We will calculate intraclass correlation coefficients with one-way random-effects models. ${ }^{84}$ We have examined reliability in both USA and international cohorts ${ }^{2637}$ and have found it acceptable to date. If reliability of any test is inferior to that seen in other cohorts, likely reasons for reduced reliability will be examined, and test-retest procedures will be repeated with the next 50 enrolled participants.

\section{Validity}

Because there is no 'gold standard' cognitive assessment in Botswana to which we can compare the PennCNB, we will use several procedures to provide evidence of validity in this setting. To examine concurrent validity, we will compare mean scores and effects of sex and age in Botswana HUU youth to US normative samples to determine whether expected effects are present. Although scores may be lower in youth from Botswana compared with US youth, especially on speeded measures, ${ }^{85}$ overall effects of age and sex should show similar patterns, which are well characterised in previous work. ${ }^{49}$ To examine predictive validity, we will compare CNB scores to the gold standard assessment classifications and evaluate its discriminability between children with and without clinically significant impairment. To assess the ability of the CNB to identify cases and controls, we will calculate classification accuracy statistics (eg, sensitivity, specificity, 
positive predictive value) and area under the receiver operating characteristic curves. To maximise generalisability of findings, all prediction results will be crossvalidated by leave-one-out and bootstrapped split-half approaches.

We will conduct factor analyses to determine whether the structural validity of the battery is consistent with theory and past work. We will compare the PennCNB factor structure across groups to test for measurement invariance, as we have done previously. ${ }^{31} 38$ We will examine group differences in cognitive performance on the PennCNB, comparing overall and individual test performance using both age-corrected scores and rates of impairment within each domain, using a well-validated deficit score approach. ${ }^{86}$ Neuropsychological norms will be generated for each 2-year age bin. Comparisons among HIV+, HEU and HUU groups will be conducted using both multiple regression (for continuous scores) and logistic regression (for impairment outcomes), with comparison of adjusted and unadjusted models to assess the influence of covariates (eg, sociodemographic factors). We will also use multivariate normative comparisons $^{87-89}$ to account for the covariances among tests when assessing deviation of scores on any given test, avoiding a multiple-testing problem. It is expected that, compared with HUU, children with HIV will demonstrate significantly worse overall CNB performance, with the greatest impairment in domains most consistently shown to be impaired in paediatric HIV, including executive functioning (eg, working memory) and attention and processing speed, indicating convergent validity. We also expect that children with HIV will demonstrate smaller deficits on subtests measuring domains with less consistent evidence for deficits (eg, spatial processing).

After the parents of 300 study participants have completed the PSC, classification accuracy statistics will assess the ability of the PSC as a whole and individual PSC questions to identify children with cognitive impairment on the PennCNB. Questions that lack association with PennCNB classifications will be considered for removal. In-depth semistructured interviews of parents of children who had abnormal CNB testing will help to identify new candidate questions for the 'Ntemoga Screener' tool. In addition, an outcome-neutral method of scale construction-that is, use of computerised adaptive test simulation ${ }^{70}$ - will be used to compare items selected using the two methods.

Parents of the final $\sim 200$ participants to enrol will complete the Ntemoga Screener, and associations between scores on the Ntemoga Screener and the PennCNB will be calculated. We hypothesise that the Ntemoga Screener will more readily identify children at risk for neurocognitive difficulties.

\section{ETHICS AND DISSEMINATION}

Ethical approval was granted by the Health Research and Development Division of the Botswana Ministry of
Health and Wellness, Botswana-Baylor Children's Clinical $\mathrm{COE}$, and University of Pennsylvania Institutional Review Board. Children will provide assent, and parents/guardians will provide informed consent prior to participation. Informed consent/assent materials will be presented in Setswana or English, according to participant/parental preference. Potential participants will be excluded if they have severe developmental delays that prevent them from following simple instructions or are unable to assent, or if consent cannot be obtained from an authorised parent/ guardian.

This project seeks to address the lack of validated tools to measure neurocognitive deficits among children affected by HIV in resource-limited settings, a significant public health issue. Findings from this research will be disseminated in peer-reviewed journals and scientific meetings, and we will communicate results and implications to critical stakeholders in Botswana, including regular updates to our stakeholder group.

In the future, tools developed from this study could provide practical screening and streamlined, comprehensive assessments that could be widely implemented in resource-limited settings to identify children with neurocognitive deficits, who could then be targeted for specific rehabilitation programmes. Thus, the proposed study could lay the groundwork for increased access to paediatric cognitive assessments in resource-limited settings. On-going mixed-methods research led by AEVP is obtaining implementation guidance by eliciting stakeholder perspectives in the medical and educational sectors in Botswana to identify factors likely to influence the success of implementing the PennCNB. These data will inform implementation strategies for the effective use of the PennCNB to support child health and wellness in Botswana.

\section{Author affiliations}

${ }^{1}$ Department of Psychiatry, University of Pennsylvania Perelman School of Medicine, Philadelphia, Pennsylvania, USA

${ }^{2}$ VISN4 Mental IIIness Research, Education, and Clinical Center, Crescenz VA Medical Center, Philadelphia, Pennsylvania, USA

${ }^{3}$ The Children's Hospital of Philadelphia, Philadelphia, Pennsylvania, USA

${ }^{4}$ Pediatrics and Epidemiology, University of Pennsylvania Perelman School of Medicine, Philadelphia, Pennsylvania, USA

${ }^{5}$ Botswana-Baylor Children's Clinical Centre of Excellence, Gaborone, Botswana ${ }^{6}$ Baylor College of Medicine, Gaborone, Botswana

Acknowledgements The authors wish to thank our Stakeholder Group in Botswana who provided invaluable guidance and support for this project.

Contributors Study concept and design: JCS, EDL, AEVP, TMM, RCG, MM. Drafting of the manuscript: JCS, EDL, AEVP, TMM. Critical revision of the manuscript for important intellectual content: JCS, EDL, AEVP, AMP, LN, TMM, RCG, OP, OT, MM, KR, JC. Obtained funding: JCS, EDL. Technical, software and material support: AMP, LN, OP, OT, MM, KR, JCS. Study supervision: JCS, EDL.

Funding This work was supported by the NIH (R01 HD095278). TMM is supported by the Lifespan Brain Institute (LiBI) of the University of Pennsylvania and the Children's Hospital of Philadelphia. Indirect support was received from two NIHfunded centers at Penn, the Penn Center for AIDS Research (CFAR; P30 Al045008) and the Penn Mental Health AIDS Research Center (PMHARC; P30 MH097488). The funding sources had no role in the design of the study; collection, analysis and interpretation of data; or in writing the manuscript. 
Competing interests None declared.

Patient and public involvement Patients and/or the public were involved in the design, or conduct, or reporting, or dissemination plans of this research. Refer to the Methods section for further details.

Patient consent for publication Not required.

Provenance and peer review Not commissioned; peer reviewed for ethical and funding approval prior to submission.

Open access This is an open access article distributed in accordance with the Creative Commons Attribution Non Commercial (CC BY-NC 4.0) license, which permits others to distribute, remix, adapt, build upon this work non-commercially, and license their derivative works on different terms, provided the original work is properly cited, appropriate credit is given, any changes made indicated, and the use is non-commercial. See: http://creativecommons.org/licenses/by-nc/4.0/.

\section{ORCID iD}

J Cobb Scott http://orcid.org/0000-0001-6538-9043

\section{REFERENCES}

1 Joint United Nations Programme on HIV/AIDS. Children and HIV: the fact sheet. Geneva, Switzerland, 2016.

2 Strehlau R, Kuhn L, Abrams EJ, et al. Hiv-Associated neurodevelopmental delay: prevalence, predictors and persistence in relation to antiretroviral therapy initiation and viral suppression. Child Care Health Dev 2016;42:881-9.

3 Patel K, Ming X, Williams PL, et al. Impact of HAART and CNSpenetrating antiretroviral regimens on HIV encephalopathy among perinatally infected children and adolescents. AIDS 2009;23:1893-901.

4 Cohen S, Ter Stege JA, Geurtsen GJ, et al. Poorer cognitive performance in perinatally HIV-infected children versus healthy socioeconomically matched controls. Clin Infect Dis 2015;60:1111-9.

5 Hoare $\mathrm{J}$, Jacqueline $\mathrm{H}$, Westgarth-Taylor $\mathrm{J}$, et al. A diffusion tensor imaging and neuropsychological study of prospective memory impairment in South African HIV positive individuals. Metab Brain Dis 2012;27:289-97.

6 Koekkoek S, de Sonneville LMJ, Wolfs TFW, et al. Neurocognitive function profile in HIV-infected school-age children. Eur $\mathrm{J}$ Paediatr Neurol 2008;12:290-7.

7 Walker SY, Pierre RB, Christie CDC, et al. Neurocognitive function in HIV-positive children in a developing country. Int $J$ Infect Dis 2013;17:e862-7.

8 Ruel TD, Boivin MJ, Boal HE, et al. Neurocognitive and motor deficits in HIV-infected Ugandan children with high CD4 cell counts. Clin Infect Dis 2012;54:1001-9.

9 Llorente AM, Brouwers P, Leighty R, et al. An analysis of select emerging executive skills in perinatally HIV-1-infected children. Appl Neuropsychol Child 2014;3:10-25.

10 Puthanakit T, Ananworanich J, Vonthanak S, et al. Cognitive function and neurodevelopmental outcomes in HIV-infected children older than 1 year of age randomized to early versus deferred antiretroviral therapy: the predict neurodevelopmental study. Pediatr Infect Dis J 2013;32:501-8

11 Smith R, Chernoff M, Williams PL, et al. Impact of HIV severity on cognitive and adaptive functioning during childhood and adolescence. Pediatr Infect Dis J 2012;31:592-8.

12 Ezeamama AE, Kizza FN, Zalwango SK, et al. Perinatal HIV status and executive function during school-age and adolescence: a comparative study of long-term cognitive capacity among children from a high HIV prevalence setting. Medicine 2016:95:e3438.

13 Brackis-Cott E, Kang E, Dolezal C, et al. The impact of perinatal HIV infection on older school-aged children's and adolescents' receptive language and word recognition skills. AIDS Patient Care STDS 2009;23:415-21.

14 Phillips N, Amos T, Kuo C, et al. Hiv-Associated cognitive impairment in perinatally infected children: a meta-analysis. Pediatrics 2016:138.

15 Nichols SL, Montepiedra G, Farley JJ, et al. Cognitive, academic, and behavioral correlates of medication adherence in children and adolescents with perinatally acquired HIV infection. J Dev Behav Pediatr 2012;33:298-308.

16 Ananworanich J, Melvin D, Amador JTR, et al. Neurocognition and quality of life after reinitiating antiretroviral therapy in children randomized to planned treatment interruption. AIDS 2016;30:1075-81.

17 Linn K, Fay A, Meddles K, et al. Hiv-Related cognitive impairment of orphans in Myanmar with vertically transmitted HIV taking antiretroviral therapy. Pediatr Neurol 2015;53:485-90.
18 Hoare J, Fouche J-P, Phillips N, et al. White matter micro-structural changes in ART-naive and ART-treated children and adolescents infected with HIV in South Africa. AIDS 2015;29:1793-801.

19 Lazarus JR, Rutstein RM, Lowenthal ED. Treatment initiation factors and cognitive outcome in youth with perinatally acquired HIV infection. HIV Med 2015;16:355-61.

20 Foster CJ, Biggs RL, Melvin D, et al. Neurodevelopmental outcomes in children with HIV infection under 3 years of age. Dev Med Child Neurol 2006;48:677-82.

21 Tran LT, Roos A, Fouche J-P, et al. White matter microstructural integrity and neurobehavioral outcome of HIV-exposed uninfected neonates. Medicine 2016;95:e2577.

22 Desmonde S, Goetghebuer T, Thorne C, et al. Health and survival of HIV perinatally exposed but uninfected children born to HIV-infected mothers. Curr Opin HIV AIDS 2016;11:465-76.

23 Donald KA, Walker KG, Kilborn T, et al. Hiv encephalopathy: pediatric case series description and insights from the clinic coalface. AIDS Res Ther 2015;12:2.

24 Kamminga J, Cysique LA, Lu G, et al. Validity of cognitive screens for HIV-associated neurocognitive disorder: a systematic review and an informed screen selection guide. Curr HIVIAIDS Rep 2013;10:342-55.

25 Antinori A, Arendt G, Becker JT, et al. Updated research nosology for HIV-associated neurocognitive disorders. Neurology 2007;69:1789-99.

26 Gur RC, Ragland JD, Moberg PJ, et al. Computerized neurocognitive scanning: I. methodology and validation in healthy people. Neuropsychopharmacology 2001;25:766-76.

27 Gur RC, Richard J, Hughett P, et al. A cognitive neurosciencebased computerized battery for efficient measurement of individual differences: standardization and initial construct validation. $J$ Neurosci Methods 2010;187:254-62.

28 Gur RC, Richard J, Calkins ME, et al. Age group and sex differences in performance on a computerized neurocognitive battery in children age 8-21. Neuropsychology 2012;26:251-65.

29 Aliyu MH, Calkins ME, Swanson CL, et al. Project among AfricanAmericans to explore risks for schizophrenia (PAARTNERS): recruitment and assessment methods. Schizophr Res 2006;87:32-44.

30 Almasy L, Gur RC, Haack K, et al. A genome screen for quantitative trait loci influencing schizophrenia and neurocognitive phenotypes. Am J Psychiatry 2008;165:1185-92.

31 Moore TM, Reise SP, Gur RE, et al. Psychometric properties of the Penn computerized neurocognitive battery. Neuropsychology 2015;29:235-46.

32 Roalf DR, Ruparel K, Gur RE, et al. Neuroimaging predictors of cognitive performance across a standardized neurocognitive battery. Neuropsychology 2014;28:161-76.

33 Satterthwaite TD, Wolf DH, Erus $\mathrm{G}$, et al. Functional maturation of the executive system during adolescence. J Neurosci 2013;33:16249-61.

34 Gur RC, Ragland JD, Resnick SM, et al. Lateralized increases in cerebral blood flow during performance of verbal and spatial tasks: relationship with performance level. Brain Cogn 1994;24:244-58.

35 Gur RC, Alsop D, Glahn D, et al. An fMRI study of sex differences in regional activation to a verbal and a spatial task. Brain Lang 2000;74:157-70.

36 Heaton RK, Franklin DR, Ellis RJ, et al. Hiv-Associated neurocognitive disorders before and during the era of combination antiretroviral therapy: differences in rates, nature, and predictors. $J$ Neurovirol 2011:17:3-16.

37 Ibrahim I, Tobar S, Elassy M, et al. Practice effects distort translational validity estimates for a neurocognitive battery. J Clin Exp Neuropsychol 2015;37:530-7.

38 Moore TM, Gur RC, Thomas ML, et al. Development, administration, and structural validity of a brief, computerized neurocognitive battery. Assessment 2017:1073191116689820.

39 Bhatia T, Mazumdar S, Wood J, et al. A randomised controlled trial of adjunctive yoga and adjunctive physical exercise training for cognitive dysfunction in schizophrenia. Acta Neuropsychiatr 2017:29:102-14.

40 Basner M, Savitt A, Moore TM, et al. Development and validation of the cognition test battery for spaceflight. Aerosp Med Hum Perform 2015;86:942-52.

41 Garrett-Bakelman FE, Darshi M, Green SJ, et al. The NASA twins study: a multidimensional analysis of a year-long human spaceflight.. Science 2019:364.

42 Goldenberg PC, Calkins ME, Richard J, et al. Computerized neurocognitive profile in young people with 22q11.2 deletion syndrome compared to youths with schizophrenia and atrisk for psychosis. Am J Med Genet B Neuropsychiatr Genet 2012;159B:87-93. 
43 Weinberger R, Yi J, Calkins M, et al. Neurocognitive profile in psychotic versus nonpsychotic individuals with 22q11.2 deletion syndrome. Eur Neuropsychopharmacol 2016;26:1610-8.

$44 \mathrm{Yi} \mathrm{JJ}$, Calkins ME, Tang SX, et al. Impact of psychiatric comorbidity and cognitive deficit on function in 22q11.2 deletion syndrome. $J$ Clin Psychiatry 2015;76:e1262-70.

45 Mekori-Domachevsky E, Guri Y, Yi J, et al. Negative subthreshold psychotic symptoms distinguish 22q11.2 deletion syndrome from other neurodevelopmental disorders: a two-site study. Schizophr Res 2017:188:42-9.

46 Hartung EA, Kim JY, Laney N, et al. Evaluation of Neurocognition in youth with CKD using a novel computerized neurocognitive battery. Clin J Am Soc Nephrol 2016;11:39-46.

47 Calkins ME, Merikangas KR, Moore TM, et al. The Philadelphia neurodevelopmental cohort: constructing a deep phenotyping collaborative. J Child Psychol Psychiatry 2015;56:1356-69.

48 Campbell MM, Sibeko G, Mall S, et al. The content of delusions in a sample of South African Xhosa people with schizophrenia. BMC Psychiatry 2017:17.

49 Swagerman SC, de Geus EJC, Kan K-J, et al. The computerized neurocognitive battery: validation, aging effects, and heritability across cognitive domains. Neuropsychology 2016;30:53-64.

50 Vatss S, Mehar $\mathrm{H}$, Bhatia T, et al. Patterns of tobacco consumption among Indian men with schizophrenia compared to their male siblings. Psychiatry Investig 2012;9:245-51.

$51 \mathrm{Yi}$ JJ, Weinberger R, Moore TM, et al. Performance on a computerized neurocognitive battery in 22q11.2 deletion syndrome: a comparison between US and Israeli cohorts. Brain Cogn 2016;106:33-41.

$52 \mathrm{Ji} \mathrm{X}$, Cui N, Liu J. Neurocognitive function is associated with serum iron status in early adolescents. Biol Res Nurs 2017:19:269-77.

53 Stephenson J. "New Dawn" for Botswana. JAMA 2004;292:2565.

54 Jellinek MS, Murphy JM, Burns BJ. Brief psychosocial screening in outpatient pediatric practice. J Pediatr 1986;109:371-8.

55 Lowenthal E, Lawler K, Harari N, et al. Validation of the pediatric symptom checklist in HIV-infected Batswana. J Child Adolesc Ment Health 2011;23:17-28.

56 Lowenthal E, Lawler K, Harari N, et al. Rapid psychosocial function screening test identified treatment failure in HIV+ African youth. AIDS Care 2012;24:722-7.

57 Lowenthal ED, Marukutira T, Tshume O, et al. Prediction of HIV virologic failure among adolescents using the pediatric symptom checklist. AIDS Behav 2015;19:2044-8.

58 Llorente AM, Turcich M, Lawrence KA. Differences in neuropsychological performance associated with ethnicity in children with HIV-1 infection: preliminary findings. Appl Neuropsychol 2004; 11:47-53.

59 Ravindran OS, Rani MP, Priya G. Cognitive deficits in HIV infected children. Indian J Psychol Med 2014;36:255-9.

60 Riccio CA, Reynolds CR, Lowe P, et al. The continuous performance test: a window on the neural substrates for attention? Arch Clin Neuropsychol 2002;17:235-72.

61 Bachman P, Reichenberg A, Rice P, et al. Deconstructing processing speed deficits in schizophrenia: application of a parametric digit symbol coding test. Schizophr Res 2010;118:6-11.

62 Gorenstein EE, Mammato CA, Sandy JM. Performance of inattentiveoveractive children on selected measures of prefrontal-type function. J Clin Psychol 1989;45:619-32.

63 Thomas ML, Brown GG, Gur RC, et al. Parallel psychometric and cognitive modeling analyses of the Penn face memory test in the army study to assess risk and resilience in Servicemembers. $J$ Clin Exp Neuropsychol 2013;35:225-45.

64 Calkins ME, Gur RC, Ragland JD, et al. Face recognition memory deficits and visual object memory performance in patients with schizophrenia and their relatives. Am J Psychiatry 2005;162:1963-6.

65 Kurtz MM, Ragland JD, Moberg PJ, et al. The Penn conditional exclusion test: a new measure of executive-function with alternate forms of repeat administration. Arch Clin Neuropsychol 2004;19:191-201.

66 Kurtz MM, Wexler BE, Bell MD. The Penn conditional exclusion test (PCET): relationship to the Wisconsin card sorting test and work function in patients with schizophrenia. Schizophr Res 2004:68:95-102.

67 Tsuchida A, Fellows LK. Lesion evidence that two distinct regions within prefrontal cortex are critical for $n$-back performance in humans. J Cogn Neurosci 2009;21:2263-75.

68 Ragland JD, Turetsky BI, Gur RC, et al. Working memory for complex figures: an fMRI comparison of letter and fractal n-back tasks. Neuropsychology 2002;16:370-9.

69 Miskin N, Thesen T, Barr WB, et al. Prefrontal lobe structural integrity and TRAIL making test, part B: converging findings from surfacebased cortical thickness and voxel-based lesion symptom analyses. Brain Imaging Behav 2016;10:675-85.

70 Moore TM, Scott JC, Reise SP, et al. Development of an abbreviated form of the Penn line orientation test using large samples and computerized adaptive test simulation. Psychol Assess 2015;27:955-64.

71 Allen MD, Fong AK. Clinical application of standardized cognitive assessment using fMRI. I. matrix Reasoning. Behav Neurol 2008;20:127-40.

72 Bilker WB, Hansen JA, Brensinger CM, et al. Development of abbreviated nine-item forms of the raven's standard progressive matrices test. Assessment 2012;19:354-69.

73 Da Silva FN, Irani F, Richard J, et al. More than just tapping: index finger-tapping measures procedural learning in schizophrenia. Schizophr Res 2012;137:234-40.

74 van Widenfelt BM, Treffers PDA, de Beurs E, et al. Translation and cross-cultural adaptation of assessment instruments used in psychological research with children and families. Clin Child Fam Psychol Rev 2005;8:135-47.

75 Guillemin F, Bombardier C, Beaton D. Cross-Cultural adaptation of health-related quality of life measures: literature review and proposed guidelines. J Clin Epidemiol 1993;46:1417-32.

76 Nasreddine ZS, Phillips NA, Bédirian V, et al. The Montreal cognitive assessment, MoCA: a brief screening tool for mild cognitive impairment. J Am Geriatr Soc 2005;53:695-9.

77 Hacker KA, Williams S, Myagmarjav E, et al. Persistence and change in pediatric symptom checklist scores over 10 to 18 months. Acad Pediatr 2009;9:270-7.

78 Pagano ME, Cassidy LJ, Little M, et al. Identifying psychosocial dysfunction in school-age children: the pediatric symptom checklist as a self-report measure. Psychol Sch 2000;37:91-106.

79 Jellinek M, Evans N, Knight RB. Use of a behavior checklist on a pediatric inpatient unit. J Pediatr 1979;94:156-8.

80 Reijneveld SA, Vogels AGC, Hoekstra F, et al. Use of the pediatric symptom checklist for the detection of psychosocial problems in preventive child healthcare. BMC Public Health 2006;6:197.

81 Murphy M. Personal communication regarding use of the pediatric symptom checklist to screen for developmental disabilities 2017.

82 Olson RS, Urbanowicz RJ, Andrews PC, et al. Automating biomedical data science through tree-based pipeline optimization. In: European conference on the applications of evolutionary computation. Springer, 2016: 123-37.

83 Zinbarg RE, Revelle W, Yovel I, et al. Cronbach's $\alpha$, Revelle's $\beta$, and Mcdonald's $\omega \mathrm{H}$ : their relations with each other and two alternative conceptualizations of reliability. Psychometrika 2005;70:123-33.

84 Shrout PE, Fleiss JL. Intraclass correlations: uses in assessing rater reliability. Psychol Bull 1979;86:420-8.

85 Robertson K, Liner J, Heaton R. Neuropsychological assessment of HIV-infected populations in international settings. Neuropsychol Rev 2009;19:232-49.

86 Carey CL, Woods SP, Gonzalez R, et al. Predictive validity of global deficit scores in detecting neuropsychological impairment in HIV infection. J Clin Exp Neuropsychol 2004;26:307-19.

87 Agelink van Rentergem JA, Murre JMJ, Huizenga HM. Multivariate normative comparisons using an aggregated database. PLoS One 2017;12:e0173218.

88 Huizenga HM, Smeding H, Grasman RPPP, et al. Multivariate normative comparisons. Neuropsychologia 2007;45:2534-42.

89 Su T, Schouten J, Geurtsen GJ, et al. Multivariate normative comparison, a novel method for more reliably detecting cognitive impairment in HIV infection. AIDS 2015;29:547-57. 